\title{
The Infectious Vaccines against SARS-CoV-2 Already Exist
}

\author{
Ding J* \\ Retired, Integrated Electronic Systems Lab Co. Ltd., China
}

*Corresponding author: Jian Ding, Retired, Integrated Electronic Systems Lab Co. Ltd., Jinan 250100, China, Email: jiandus@163.com

\section{Review article}

Volume 5 Issue 3

Received Date: November 18, 2020

Published Date: December 07, 2020

DOI: $10.23880 / \mathrm{ijbp}-16000188$

\section{Abstract}

In allusion to the COVID-19 epidemic situation, the current key point to win the decisive victory is on how to improve the antibody prevalence, so as to achieve herd immunity and eventually eliminate the epidemic situation. Therefore, it is strongly recommended that an authoritative experimental conclusion should be given on whether the recovered patients with COVID-19 are infectivity or not. That is to say, those susceptible people who have not yet had the antibodies should be tested, in order to verify whether they would produce antibodies after close contact with the recovered patients who have had the antibodies. Of course, this is a feasible experiment. The implication that there should be a type of infectious vaccines against SARS-CoV-2, which is always around us and well within reach. In addition, it is pointed out that the state of balance where the viruses and the human immune system are opposed and can coexist is the environment they face for survival and proliferation, while the infectivity is the necessary condition. It is under such environment that the progenies of those original viruses would be just possible to be improved by human immune system, thereby evolve into the main part of the IgG antibodies. Since asymptomatic infected persons are now confirmed to transmit the viruses, so it is inferred that the Infectious Attenuated Live Vaccine against SARS-CoV-2 must be there too.

Keywords: COVID-19; Virus; Vaccine; Antibody; Infectivity; Immune

\section{Introduction}

In April 14, 2020, the WHO mentioned in the published "COVID-19 strategy update" [1] that the COVID-19 was a new disease, the viruses were rapidly spreading, and the outbreak of the epidemic situation was growing exponentially, but it had not yet proven that there was any specific therapy or vaccine.

If the COVID-19 epidemic is divided into two halves, then whether strategically or tactically, we should try our best to be reducing mortality, which is the common ground of the two. As for the difference, the first half was a hasty response, we must take strict precautions in order to curb the spread of SARS-CoV-2 and slow down the outbreak of a pandemic. The purpose was to buy time and as much as possible to reduce the loss, in order to waiting for the safe and effective vaccines and treatments to come into being. And now it has entered the second half, the key point to win the decisive victory is on how to improve the antibody prevalence, so as to achieve herd immunity and the purpose of eventually to eliminate epidemic situation. It should be said that this is also in line with the WHO's strategic intention against COVID-19.

This article intends to point out that before the safe and effective vaccines to come into being, an authoritative experimental conclusion should be given on whether the recovered patients from COVID-19 are with infectivity or not. That is to say, those susceptible people who have not yet had the antibodies should be tested, in order to verify whether they would produce antibodies after close contact with the recovered patients who have had the antibodies.

Of course, this is a feasible experiment. The implication that there should be a type of infectious vaccines against SARS-CoV-2, which is always around us and well within 


\section{International Journal of Biochemistry \& Physiology}

reach. If not taken seriously, or even dismissive, how can we face the souls of the deceased of the hundreds of thousands in heaven? Besides, for those recovered patients who already have the antibodies, whether they are with infectivity or not, if relevant institutions and experts including the WHO would not give an authoritative experimental conclusion, then it is impossible to fully understand the ecological environment of SARS-CoV-2, which in itself is a strategic omission.

\section{The background of the omission is the idea}

Foods that contributing to the human body are usually considered to contain nutrients, while those that can cause harm are considered to be toxic. Therefore, at the micro level, since it is called a virus, it certainly means that it is harmful to human body. But even within the same category of viruses, their toxicity would also be quite different, just like everyone's ability can also change with the environment and time.

People will change their behavior after being educated, restricted or punished, and the same is true for viruses. This is the law of nature, namely survival of the fittest. And also it should be noted that the toxicity and infectivity of the virus are two different concepts and should not be confused together. In other words, the weakening of the toxicity of the virus does not mean that its infectivity is also weakening.

In terms of the recovered patients with COVID-19, were the SARS-CoV-2 viruses which infected before recovery killed by the antibody produced by the human immune system, or they evolved into that the two could coexist peacefully? There has been no clear answer. In the face of such problem, I looked up relevant discourses of today's authoritative organizations or experts on the Internet. As for the results there were two, either avoiding answering, or passing by vague words. This means that the problem has touched the blind area of their cognition.

For example, in response to such a problem, the Chinese respiratory disease specialist Academician Zhong Nanshan once stated [2] that the antibodies had a strong comprehensive killing effect against SARS-CoV-2. Among them, the meaning of "comprehensive killing" was ambiguous. Although it could also be understood as covering the latter, but the feeling left to people was to focus on the former. Namely the antibody not only killed the SARS-CoV-2, but also enabled the human body to have a certain degree of immunity.

This also represents the mainstream idea of relevant organizations or infectious disease experts today. In the face of the tragic COVID-19 epidemic for more than half a year, they sometimes had to use the plasma of recovered patients to treat the patients, which was of course because therein to has contained antibodies against SARS-CoV-2. But the strange thing was that why couldn't they further broaden thinking to use relevant serology surveillance to verify whether the recovered patients were with infectivity? The reason for this was still on the idea that the virus should be "comprehensively killed", rather than tightly grasping on the main contradiction that was the infectivity, then riding on the momentum according to the ecological environment of SARS-CoV-2, so that let those viruses that had evolved into coexisting with the human body for our use.

\section{The Focus is on how to improve the Antibody Prevalence}

The so-called antibody prevalency, also known as seroprevalence, here mainly refers to the proportion that the people who have been positive with the IgG antibodies against SARS-CoV-2 in the total number of the tested population. Viewing from a statistical perspective, it can be considered as the proportion that all people who have been infected with the SARS-CoV-2 in the total population of the region. From this it can be calculated how many susceptible people who have not yet had the antibodies are in this region.

As far as recovered patients were concerned, they had gone through a rigorous nucleic acid testing (RT-PCR) procedure, and confirmed that the result was negative, namely believed that there was no virus replication. In view of this, Academician Zhong Nanshan asserted: "the antibodies have a strong comprehensive killing effect against the SARS-CoV-2. For most people, the chance of repeated infection is small." Moreover, according to the previous large number of clinical experience, he also introduced: "Basically did not see the people who had been in close contact with the recurrence positive patients were infected [2]."

Looking back on January 20th, Academician Zhong Nanshan confirmed on behalf of his country that the new type of coronavirus (SARS-CoV-2) could be passed on from person to person [3]. It can be seen that most people's understanding for this conclusion was still in a fuzzy state at that time. And with the development of the epidemic situation, by April 6th, the State Council issued to affirm [4] that the asymptomatic infected persons were with infectivity, and there was a risk of transmission. In view of this process, now if transposition thinking, is not this precisely SARS-CoV-2 that forces us to understand its infectivity? It seems to suggest that you need to breathe, but I can infect, you can't kill me as I can also mutate, so the coexistence is the hard truth.

In order to achieve herd immunity and the purpose of eventually eliminating the epidemic situation, the current strategic focus is on how to improve the antibody prevalence. Therefore, using relevant serology surveillance as evidence 


\section{International Journal of Biochemistry \& Physiology}

to confirm whether the recovered patients with COVID-19 are with infectivity or not, is a key step to understand the whole ecological environment of SARS-CoV-2 from a macro perspective. That is to say, those susceptible people who have not yet had the antibodies should be tested, in order to verify whether they would produce antibodies after close contact with the recovered patients who have had the antibodies. Once the antibodies are found among the susceptible people who did not yet have the antibodies, it has proved that the recovered patients, whether they are recurrence positive patients or not, all are with infectivity.

According to the WHO's global strategy on COVID-19, many countries have successively begun the testing work for the antibody prevalence against SARS-CoV-2 at the turn of the spring and summer of this year. After that, the chief scientist at WHO, Dr. Soumya Swaminathan said that the number of people infected often was about 10 times the number of people who actually was diagnosed as cases. According to this authoritative data, it can be known that among them the mild or asymptomatic infected people accounted for an overwhelming majority, and the death rate of infected people has dropped about 10 times along with it, too [5].

\section{The Vaccine with Infectivity Must Exist}

The first example [6] was in Wuhan, where was the hardest hit area of the COVID-19 epidemic situation in China, and the measured antibody prevalence was about $3.8 \%$. And Beijing, the capital of China, has been the key protective zone in the COVID-19 epidemic situation. It can be inferred that the antibody prevalence there should be far less than $3.8 \%$. In other words, almost all of the population in this city should be susceptible people who have not yet had antibodies. Although the government has taken various effective protective measures, but these people seemed to lie in a tent that must be breathable, in the face of all-pervasive viruses (SARS-CoV-2), it would be really impossible to defend effectively!

The second example [7] was the city of Bergamo in the Lombardy region of northern Italy. As the hardest hit area of the country's COVID-19 epidemic situation, according to the Italian health department citing a sample survey, among the 9,965 residents of Bergamo who underwent random antibody testing between April 23 and June 3, about 57\% of the tested people's serum antibody was positive.

The total population of Bergamo city was about 120000 . According to the official statistics at the time and considering the existence of additional death factors attributable to COVID-19, so using the data of 13,609 infected individuals with SARS-CoV-2 (including its surrounding area), it could be calculated that the infection rate was about $11 \%$. If based on the antibody prevalence was $57 \%$ to calculate, it was exactly in line with the WHO's [8] that about $80 \%$ of the infected individuals were the mild or asymptomatic patients. In general, it can be considered as having herd immunity when the antibody prevalence is greater than $60 \%$. But this was at the cost of the lives of about 3,000 people, not the outcome we wanted. It was a failure case.

In addition, only slightly more than $30 \%$ of the 10,404 medical workers tested in the region were found to have antibodies. This meant that those protective equipment and rules were like a double-edged sword, which could not only slow down the COVID-19 pandemic, also limited the infection rate of the mild or asymptomatic patients, namely hindered in the improvement of the antibody prevalence. So the research team in China's Chongqing previously has claimed [9] that the antibody levels of the asymptomatic patients from COVID-19 dropped faster, which should have overlooked such a factor, that was, the above-mentioned protective equipment or rules would limit their chances reinfected. This is also a key link that the WHO should evaluate and make guiding recommendations.

Now, the top priority is to improve the antibody prevalence as soon as possible. In addition to take strict precautions and wait for the vaccines, we should also broaden our thinking, and confirm as soon as possible whether the recovered patients with COVID-19 are infectivity or not. Moreover, since $80 \%$ of the infected people were mild or asymptomatic, so most viruses of cross-infection among them should belong to the category of Infectious Attenuated Live Vaccine against SARS-CoV-2. Given that the proportion was as high as $80 \%$, then it was definitely not a fortuitous event, should be an inevitable existence.

In this regard, the WHO should also be to evaluate. And sum up a set of safe and effective new therapies, so as to standardize the ecological environment of these vaccines with infectivity, thereby under the premise of reducing mortality to improve effectively the antibody prevalence. In this way, even if there is no safe and effective vaccine, it can also achieve the goal of eliminating the epidemic situation.

\section{The Viruses Tolerated by the Human Immune System}

From the perspective of medication, whether it is traditional Chinese medicine (TCM) or Western medicine, really to cure diseases at the cellular level, still needs to be executed by the body's own immune system. Except for the immune system, modern humans have not yet discovered any kind of drug that can kill some kind of viruses without harming their own body. 


\section{International Journal of Biochemistry \& Physiology}

The tenet of using medication of TCM has never thought about the cellular level, just only through macroscopic control to do the utmost to make that the human body would reach his own best state, which is the so-called a balance of Yin and Yang (or a balance of Five Elements). As for how to treat diseases at the cellular level, which has completely handled by the human body's own immune system. Such repeated adjustments, when the human body gradually approaching this optimal state and reaching a certain level, it would be considered as the medicine has taken effect and so the symptoms gone away.

And the medication used by Western medicine, it is as much as possible to hold the balance of various indicators within the human body at the micro level, such as acidbase, vitamins, blood components, etc., like which are too numerous to mention. Its purpose is more direct, that is, on the premise of ensuring that the human body is in a relatively stable state as much as possible, then to drive the immune system to treat diseases at the cellular level.

The human body is like a big society. Every cell therein has to follow the laws of nature, that is, the survival of the fittest. Therefore, every cell of the human body's own is trying its best to fight the best living conditions for itself. This means that not only do they have to fight and kill each other, but also in the process of passing on from generation to generation, with gradual blending and some variations, so as to achieve a state of balance in which they can coexist. As a result, the human body like a country, has laws and a state machine, which is the immune system. Of course, the immune system must follow the laws of nature. That is to say, its function must keep pace with the times, just like the constitution of some countries, there would be various Amendments direct at immigrants.

In reality, everything is always in the process of change. Therefore, there is only likeness but no absolute identical. As far as the SARS-CoV-2 is concerned, its progenies would mutate, that is, the toxicity and infectivity of every generation would be different. And only those progenies that can be tolerated by the human immune system, are possible to achieve the balance state of opposition and coexistence with the immune system. It is exactly the environment in which they face for survival and propagation. Of course, with the external environment changes, such as the infectivity is quarantined, which would cause the powers of the opposing sides to rise and fall, so the balance state be also changed. And those progenies that lack power or cannot be tolerated by the human immune system will eventually be wiped out, because they violate the natural law of the survival of the fittest. It can be seen that as far as this kind of viruses belonging to the non-cellular microorganisms that survive by replication were concerned, the infectivity is a necessary condition that they can multiply down. This should be an objective law with universality.

\section{Conclusion and Inference}

Therefore, after repeated filtration by the human immune system, those progenies evolved from the original SARS-CoV-2 would gradually become the variants that can coexist with the immune system, which should be main part of constituting the IgG antibody against SARS-CoV-2 within human body. Just imagine, if they cannot be passed on from generation to generation, how can it be revealed that the IgG antibody against SARS-CoV-2 had been constantly positive?

Furthermore, if these progenies of SARS-CoV-2 had lost their infectivity between human bodies, then the ecological balance between them and human immune system would be broken, and their power gradually weakened in human body, so showing as the IgG antibodies against SARS-CoV-2 quickly turn into negative. This means that those people whose IgG antibodies against the progenies of a certain kind of virus are continuously positive, not only are infected persons and also with infectivity. In fact, now the asymptomatic infected persons have been affirmed as being able to transmit the virus, which is a strong example illustration.

As indicated above, for the question of whether the recovered patients from COVID-19 are with infectivity or not, whatever the results of those authoritative experiments are, we can refer to the definition of vaccine to make with further inferences. That is to say, if those authoritative experiments have affirmed that the recovered patients from COVID-19 are with infectivity, it means that the Infectious Inactivated Vaccine against SARS-CoV-2 should be regarded as existing. Although their SARS-CoV-2 nucleic acid detected by RT-PCR has been negative, and can think no virus replicated currently, but the infectivity means that the replicated viruses still exist, which would be nothing more than those progenies evolved from the original SARS-CoV-2, and have been improved by the human immune system.

Otherwise, the Infectious Attenuated Live Vaccine against SARS-CoV-2 would also be regarded as existing. Because the asymptomatic infected persons are with infectivity, and their SARS-CoV-2 nucleic acid detected by RT-PCR has been positive, so it can be considered that the viruses replicated still exist. Fortunately, the antibodies against SARS-CoV-2 have been produced within their bodies. Both being asymptomatic and can improving the antibody prevalence, is not that precisely the result we want? All in all, it can be concluded that the infectious vaccines against SARSCoV-2 already exist. So it should be validated and evaluated as soon as possible, in order to facilitate the formation of a set of new therapies matched with that. In the face of the 


\section{International Journal of Biochemistry \& Physiology}

epidemic situation, we do not be pessimistic, should broaden our thinking, fight with unity, and the victory will be at hand.

\section{References}

1. WHO (2020) COVID-19 strategy update.

2. Zhong NS (2020) Most recovered patients from COVID-19 have a small probability of re-infection.

3. Zhong NS (2020) The novel coronavirus has the phenomenon of passing on from person to person.

4. Qin YH (2020) State Council: the asymptomatic infected persons are with infectivity and should be managed like this.

5. Soumya Swaminathan (2020) World Health Organization
(WHO) Media Briefing on Global COVID-19 R\&D Forum July 2(55): 12 .

6. Xu X, Sun J, Nie S, Li H, Kong Y, et al. (2020) Seroprevalence of immunoglobulin $\mathrm{M}$ and $\mathrm{G}$ antibodies against SARSCoV-2 in China. Nat Med 26: 1196-1195.

7. Qian TX (2020) Italy's first herd immunity area appears, $57 \%$ of the population has antibodies against SARSCoV-2.

8. WHO (2020) Q\&A: Influenza and COVID-19 - similarities and differences.

9. Long Q, Tang X, Shi Q Li Q, Deng HJ, et al. (2020) Clinical and immunological assessment of asymptomatic SARSCoV-2 infections. Nat Med 26: 1200-1204. 